\title{
Ballistic-neutralized chamber transport of intense heavy ion beams*
}

\author{
D. V. Rose, , a, D. R. Welch, , B. V. Oliver $a$, R. E. Clark
} and A. Friedman

a Mission Research Corporation, 5001 Indian School Road, Albuquerque, NM 87110 3946, USA

b Lawrence Livermore National Laboratory, Livermore, CA, USA

Available online 31 May 2001.

\section{Abstract}

Two-dimensional particle-in-cell simulations of intense heavy ion beams propagating in an inertial confinement fusion (ICF) reactor chamber are presented. The ballisticneutralized transport scheme studied uses $4 \mathrm{GeV} \mathrm{Pb}+1$ ion beams injected into a lowdensity, gas-filled reactor chamber and the beam is ballistically focused onto an ICF target before entering the chamber. Charge and current neutralization of the beam is provided by the low-density background gas. The ballistic-neutralized simulations include stripping of the beam ions as the beam traverses the chamber as well as ionization of the background plasma. In addition, a series of simulations are presented that explore the charge and current neutralization of the ion beam in an evacuated chamber. For this vacuum transport mode, neutralizing electrons are only drawn from sources near the chamber entrance.

Author Keywords: Ion beam transport; Charge neutralization; Current neutralization

PACS classification codes: 52.58.Hm; 52.65.Rr; 52.40.Mj

\author{
Article Outline \\ 1. Introduction \\ 2. Simulation model \\ 3. Simulation results \\ 4. Beam distribution at the target plane \\ 5. Conclusions \\ Acknowledgements \\ References
}




\section{Introduction}

Numerical simulations using the particle-in-cell code LSP [1] are carried out to study propagation of intense heavy ion beams in an inertial confinement fusion (ICF) reactor chamber environment. The ballistic-neutralized transport scheme [2] calls for multiple 4 $\mathrm{GeV} \mathrm{Pb}$ ion beams to be transported and focused onto a two-sided ICF target [3]. The beams enter the target through an annular aperture. Charge and current neutralization of the beam is provided by a low-density background gas (FLiBe).

The role of electrons drawn from nearby walls and plasmas as the beam enters the reactor chamber are also examined in a series of simulations. Here, the ion beam is injected into an evacuated chamber where charge and current neutralizing electrons are only available from specific boundary sources such as nearby walls and/or localized plasmas. Radial profiles of the beam after $300 \mathrm{~cm}$ of transport are compared for both the ballisticneutralized and vacuum transport simulations.

\section{Simulation model}

A schematic of the simulation geometries used in this work are shown in Fig. 1. The simulation geometry at the top of Fig. 1 is referred to as the "standard" geometry while the bottom is referred to as the "beam-port" geometry. All simulations are twodimensional and axi-symmetric. The standard geometry is similar to previous HIF beam transport simulations [2, 4, 5, 6 and 7], while the beam-port geometry removes the metallic boundary condition upstream of the target chamber. The injected beam ions are 4 $\mathrm{GeV} \mathrm{Pb}+1(=0.2)$ and have a Gaussian transverse velocity spread of $1.9 \mathrm{keV}$ (or an unnormalized emittance of $30 \mathrm{mmmrad}$ ). The beam has a parabolic spatial and current profile in time with a peak particle current of $4 \mathrm{kA}$. The duration of the beam pulse is 8 ns. For the standard geometry simulations, the maximum beam radius at injection is $3 \mathrm{~cm}$ and the beam is focused and transported over $300 \mathrm{~cm}$ to the target location. For the beamport geometry simulations, the maximum injected beam radius is $3.2 \mathrm{~cm}$, and the beam is focused on axis, $320 \mathrm{~cm}$ from the injection plane. 


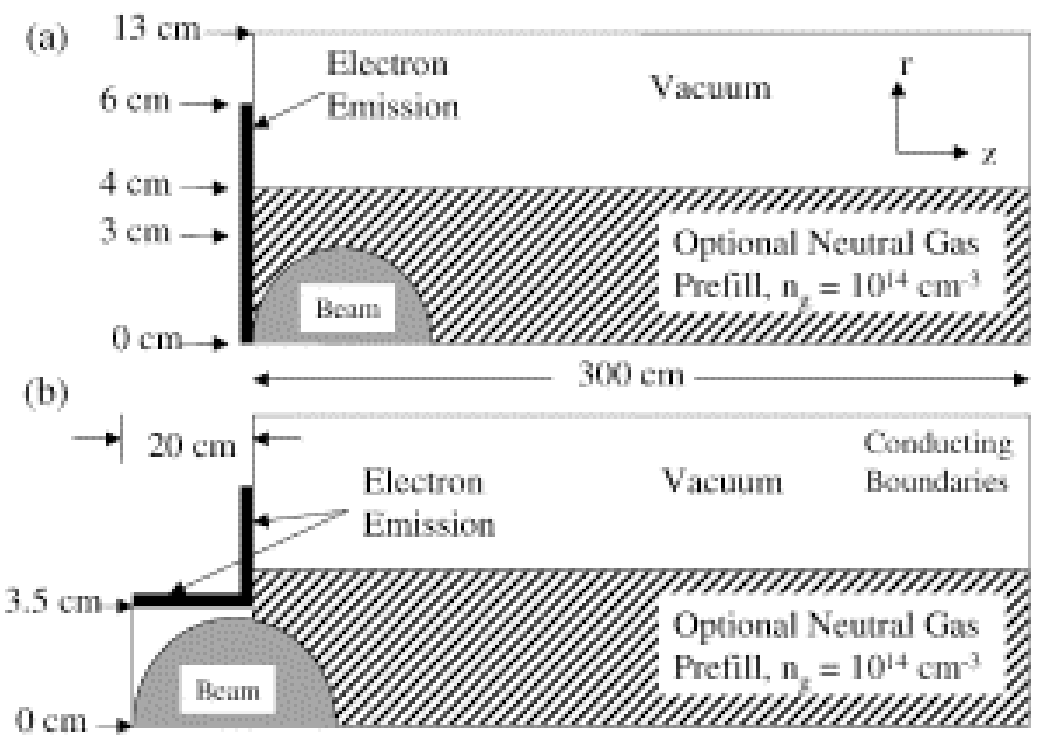

Fig. 1. Schematic of the simulation geometries, (a) "standard" and (b) "beam-port". Drawings are not to scale.

For the ballistic-neutralized simulations a Monte Carlo model is used for beam ion stripping and background gas ionization. The stripping cross-section is fixed at 2?10-16 $\mathrm{cm}-2$ for all beam charge states and the cross-sections for beam impact ionization of the $\mathrm{FLiBe}$ are taken from [6], for $10 \mathrm{GeV} \mathrm{Pb}+\mathrm{n}$ ions. The beam and chamber parameters used in this work are similar to that of Sharp et al. [7].

Electron emission from conducting surfaces is modeled with a standard space-chargelimited emission algorithm that emits sufficient electron space-charge to zero-out the electric field normal to that surface. Typically, such models also require that the electric field first reach a minimum value before the charge can be emitted. Here the field threshold is set to a very low value of $1 \mathrm{kV} / \mathrm{cm}$, enabling a ready supply of electrons, modeling a pre-ionized, local plasma. (Typical clean conducting surfaces such as aluminum require field thresholds in excess of $100 \mathrm{kV} / \mathrm{cm}$ ).

The multi-dimensional particle-in-cell code LSP [1] is used throughout in twodimensional axi-symmetric mode. The field solver used in all cases is an implicit algorithm that enables numerically stable simulations of the high current ion beam and associated high density electron distributions. In addition, LSP includes a hybrid model for simulating dense plasmas. This model has recently been tested for HIF parameter ion beams propagating in preformed background plasmas and vacuum [8]. The results of a single hybrid LSP ballistic-neutralized simulation are discussed in Section 3. 


\section{Simulation results}

A summary of the simulations is given in Table 1. The first five runs ("bn") are ballisticneutralized simulations and the remaining seven runs ("en") are vacuum transport simulations where no background gas or plasma is present in the reactor chamber. The second column of Table 1 lists the simulation geometry used (see Fig. 1), the third column lists the pre-fill gas density, ng, and the fourth column states if beam stripping (and gas ionization) were modeled in the simulation. The fifth column lists partial information about electron emission from surrounding walls. The fraction of the total beam charge, $\mathrm{fb}$, that is within $3 \mathrm{~mm}$ at $\mathrm{z}=300 \mathrm{~cm}$ is listed in the sixth column. The remaining four columns give values taken from the simulation results corresponding to the time that the beam centroid is located at $\mathrm{z} 272 \mathrm{~cm}$. The beam radius which encloses $70 \%$ of the total beam charge is given along with the azimuthal magnetic and radial electric fields at this location $[\mathrm{z}=272 \mathrm{~cm}$, and $\mathrm{rb}(70 \%)]$. The last column gives the approximate Lorentz force at this location assuming $=0.2$. The axial location of the beam centroid, $z=272 \mathrm{~cm}$, is selected because the beam head has not yet reached the conducting wall at $\mathrm{z}=300 \mathrm{~cm}$.

Table 1. Ballistic-neutralized and vacuum transport simulation parameters. "S" = standard geometry and "BP" = beam-port geometry. Run bn9 uses a pre-formed FLiBeelectron plasma of density $1013 \mathrm{~cm}-3$ throughout. Run en08 adds a pre-ionized carbonelectron plasma of density $1.5 ? 1012 \mathrm{~cm}-3$ in the beam-port section of the simulations $(\mathrm{z}=-20$ to $0 \mathrm{~cm}, \mathrm{r}=0-3.5 \mathrm{~cm})$. Runs bn $3 \mathrm{a}$ and $\mathrm{bn} 8$ are orbit calculations 
In Fig. 2, the beam ions are shown for run bn4a at four different positions. In addition to the electrons created by stripping and ionization, electrons are space-charge emitted from the left wall for $\mathrm{r} 3 \mathrm{~cm}$. At each time, the most abundant beam ion species is plotted. Also shown are curves representing the maximum beam radius as a function of axial position for cases of complete charge and current neutralization (i.e., ballistic propagation, from run bn3a) and no neutralization (from run en04). At the last two positions, the maximum beam radius is significantly larger than the ballistic radius curve, indicating incomplete charge neutralization. This conclusion is supported by the tabulated net radial force at $\mathrm{rb}(70 \%)$ and $\mathrm{z}=272 \mathrm{~cm}$. In addition, the higher charge state beam ions are accelerated out to ever larger radii by the radial electric fields.

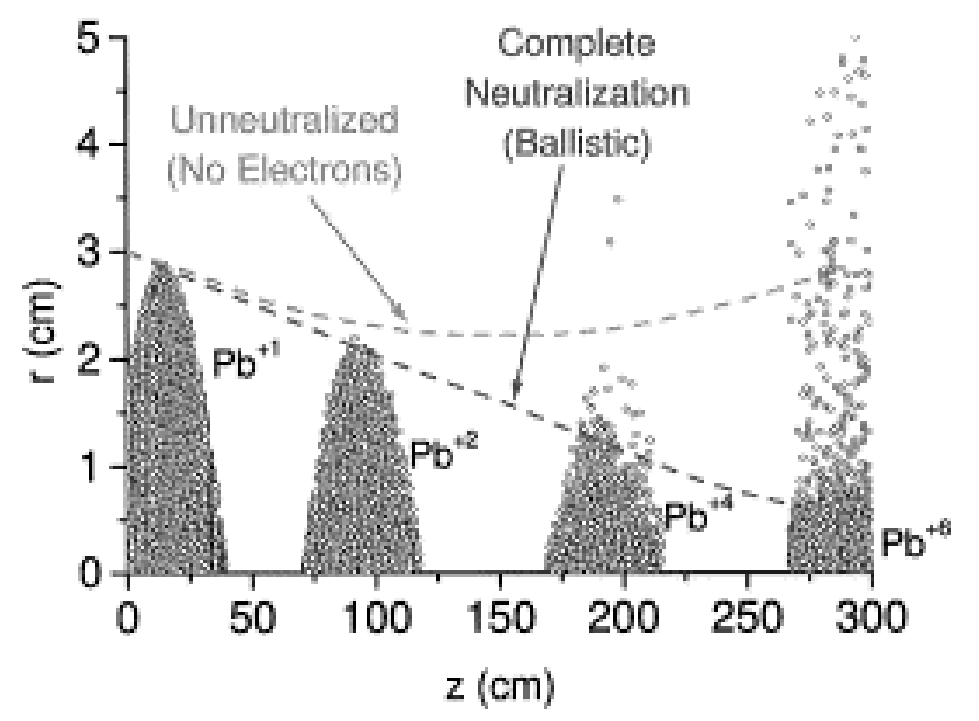

Fig. 2. Beam ions at four positions from run bn4a. At each position, the dominant beam charge state is plotted. Curves for the peak beam radius as a function of distance are shown for a simulation with no self-fields (ballistic) and for a simulation without electron sources.

When the beam-port geometry is used (run bn7a), the same basic result is obtained, although a smaller net radial force is found at $\mathrm{rb}(70 \%)$ indicating slightly better charge neutralization. The reason for this is that the inner radius of the port region in the beamport geometry effectively provides low energy electrons to the beam as it passes through the port, resulting in slightly better charge neutralization of the beam as it propagates through the chamber. This difference can be seen by examining the electron speed distributions of the wall emitted electrons in Fig. 3(b) (at $\mathrm{z}=100 \mathrm{~cm}$ ). The wall-emitted electrons form a cooler distribution in the beam-port case, drifting at a speed that is close to the beam speed. The volume created electrons (electrons created from stripping and ionization) represent the bulk of the neutralizing electrons and have a drift speed that is similar to the beam. The standard geometry simulation gives a slightly warmer electron distribution than the beam-port geometry. 


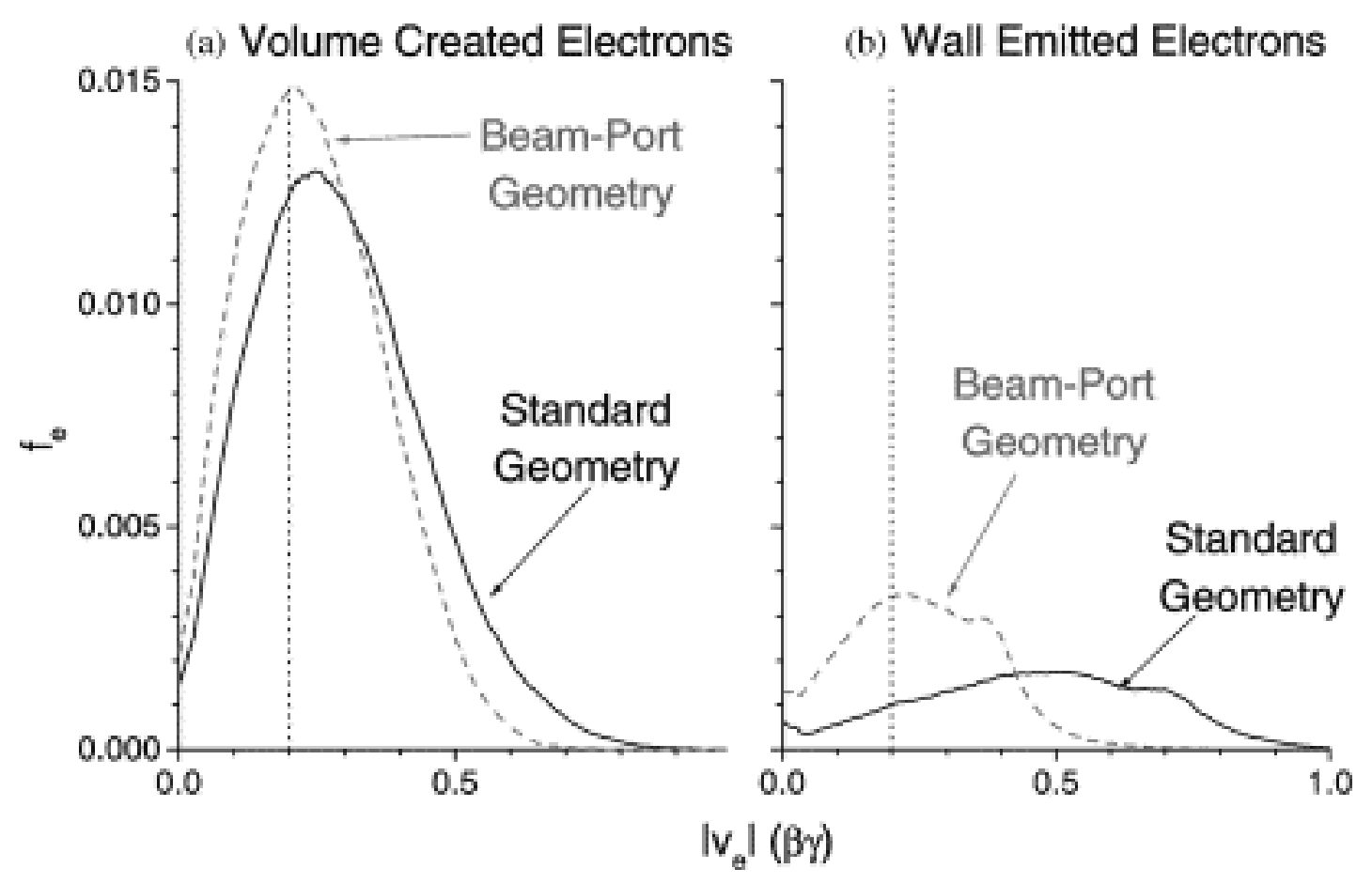

Fig. 3. Electron speed distribution functions for two ballistic-neutralized simulations (bn4a and bn7a). (a) speed distribution function for volume created electrons and (b) speed distribution for wall emitted electrons for standard and beam-port geometries at times when beam centroid is at $\mathrm{z} 100 \mathrm{~cm}$. The vertical dotted line is the beam ion speed.

Both of these ballistic-neutralized simulations (bn4a and bn7a) show evidence of energetic electrons propagating ahead of the beam, and re-entering at the back of the beam after a large radial excursion. This electron recycling phenomenon will be examined more closely in future work.

Partial pre-ionization of the background gas inside of the reactor chamber is a condition that is expected to improve neutralization of the beam. Run bn9 uses the same parameters as run bn $4 \mathrm{a}$, except that a $10 \%$ pre-ionization of the background FLiBe is established throughout the reactor chamber (about 67 times the injected beam density). Here, the plasma electrons are initialized as a $3 \mathrm{eV}$ fluid species. In the LSP hybrid model, the electron fluid particles can dynamically transit between fluid and kinetic descriptions according to a set of transition criteria [9]. Table 1 shows that the beam is highly charged, neutralized, well focused, and develops a net pinching force as it approaches the target position. Fig. 4 shows the beam at four positions during transport. Note that at only the last position does the maximum beam radius exceed the ballistic curve, indicating that the beam is well neutralized for most of the beam transport distance. 


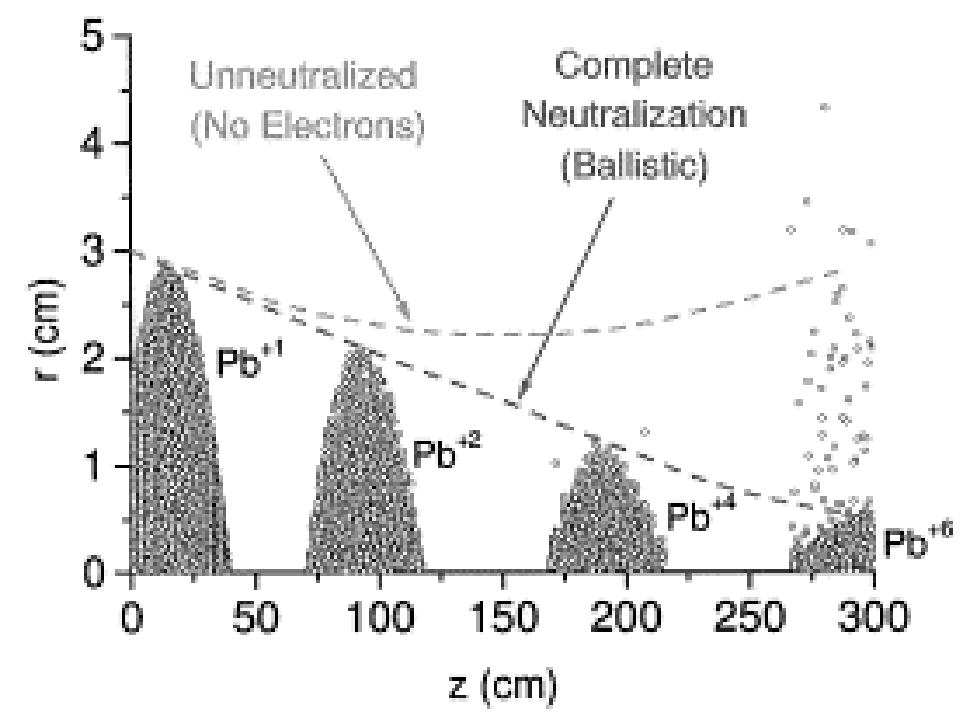

Fig. 4. Beam ions at four positions from run bn9. At each position, the dominant beam charge state is plotted. Curves for the peak beam radius as a function of distance are shown for a simulation with no self-fields (ballistic) and for a simulation without electron sources.

The vacuum transport series of simulations were carried out to explore the limits to which the converging, parabolic ion beam could be neutralized by various electron sources near the beam entrance to the reactor chamber. From Table 1, the first three "en" simulations used the standard simulation geometry, while the remaining four simulations used the beam-port geometry. Note that without any neutralizing electrons, only $2 \%$ of the beam is within $3 \mathrm{~mm}$ at $\mathrm{z}=300 \mathrm{~cm}$ in both geometries (runs en 04 and en 05 ). In run en02, which gave the largest fraction of beam charge within $3 \mathrm{~mm}$ at $\mathrm{z}=300 \mathrm{~cm}$, the only electron source was a co-injected electron beam. The electron beam used the same spatial profile, number density, current density and transverse temperature as the injected ion beam. In Fig. 5, the electron speed distribution at three different times for this run is compared with the electron speed distribution for run en03a, where the electrons are supplied only by nearby walls in the beam-port geometry. For the co-moving electron beam case, the electron distribution gradually heats as the beam radially compresses during transport. Electron losses between $\mathrm{z}=94$ and $272 \mathrm{~cm}$ are less than $1 \%$ for this case. For run en03a, the electrons trapped in the beam potential heat between $\mathrm{z}=94$ and $192 \mathrm{~cm}$, but cool slightly as beam centroid reaches $\mathrm{z}=272 \mathrm{~cm}$. For this case, some electrons escape the beam potential as the beam propagates; about $12 \%$ of the electrons escape from the beam potential between $\mathrm{z}=94$ and $272 \mathrm{~cm}$. 


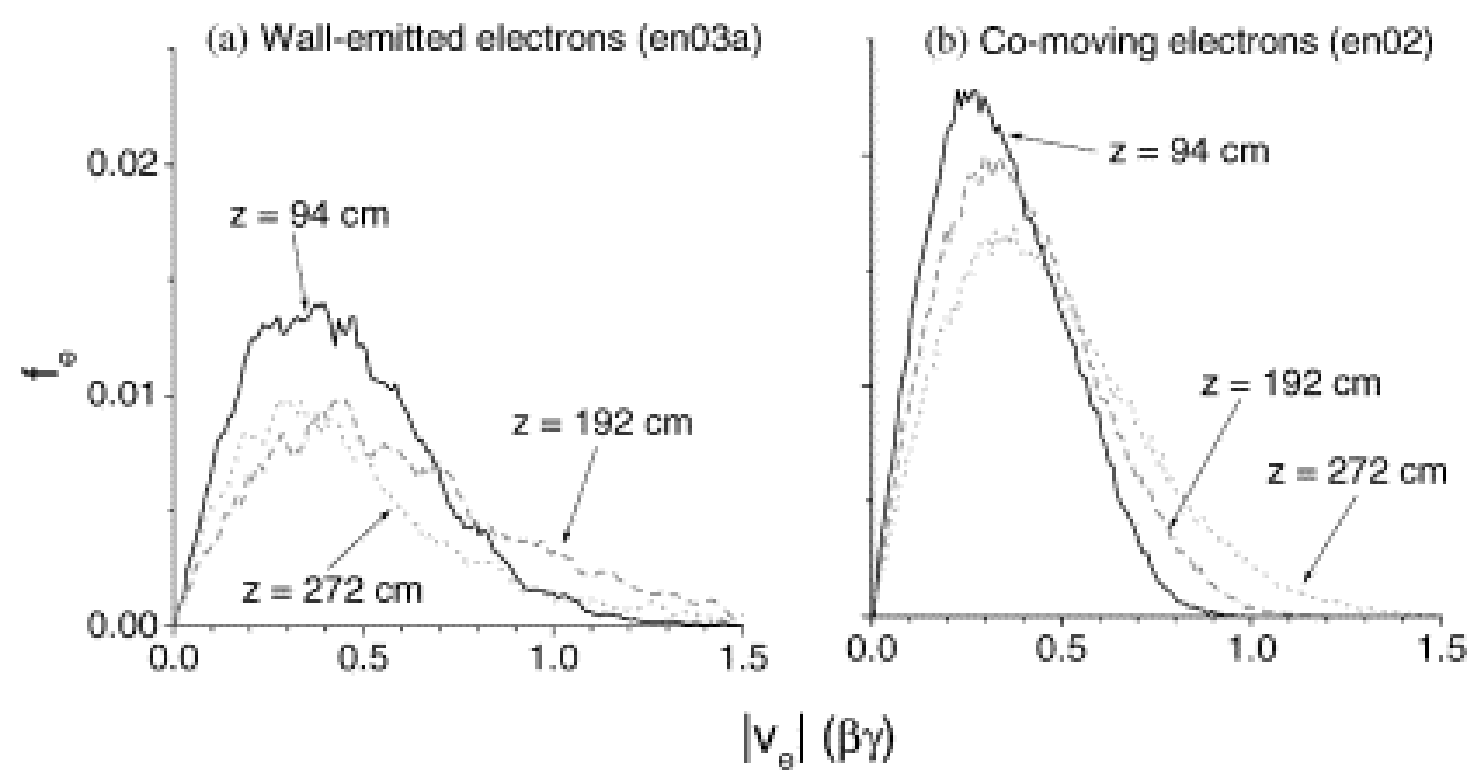

Fig. 5. Electron speed distribution functions for two vacuum transport simulations (en3a and en02). (a) Speed distribution function for wall-emitted electrons and (b) speed distribution for co-injected electrons at three times. The $\mathrm{z}$ coordinate label denotes the position of the beam centroid at each time.

Another possible mechanism to provide neutralizing electrons to the beam is to have the beam pass through a pre-formed, localized plasma outside of the reactor chamber. In run en 08 , a $3 \mathrm{eV}$ carbon-electron plasma was placed in the $3.5 \mathrm{~cm}$ radius port region of the beam-port geometry (between $\mathrm{z}=-20$ and $0 \mathrm{~cm}$ ). The pre-filled plasma density was approximately 10 times the injected beam density (np=1.5?1012 cm-3). At $\mathrm{z}=272 \mathrm{~cm}$ the beam was well neutralized by a co-moving population of $2.5 \mathrm{keV}$ electrons drifting with the beam.

\section{Beam distribution at the target plane}

In Fig. 6, the time-integrated beam number density is plotted as a function of radius for several simulations in both the standard and beam-port geometries. Note that no attempt has been made in this work to optimize the beam focal spot. The axial location that optimizes the fraction of the beam that lies within the target acceptance radius of $3 \mathrm{~mm}$ is typically less than the ballistic focal distance of $300 \mathrm{~cm}$ due to the radial space-charge expansion of the incompletely neutralized beam. From Fig. 6, the "ballistic" or orbit calculations give the highest fraction of the beam within $3 \mathrm{~mm}$ at $\mathrm{z}=300 \mathrm{~cm}$. (See Table 1 for a listing of the fraction of beam charge within $3 \mathrm{~mm}$ at $\mathrm{z}=300 \mathrm{~cm}$.) The lowest fraction within $3 \mathrm{~mm}, 0.02$, occurs for the cases without any charge or current neutralization, as expected. The ballistic-neutralized simulations for both geometries (without pre-ionization of the $\mathrm{FLiBe}$ ) give similar radial density profiles, and in both cases the higher charge-state beam ions tend to be pushed out to larger radii. Future 
simulations will look at balancing the focusing and de-focusing forces in order to optimize the fraction of the beam that is within the desired spot size of $3 \mathrm{~mm}$.
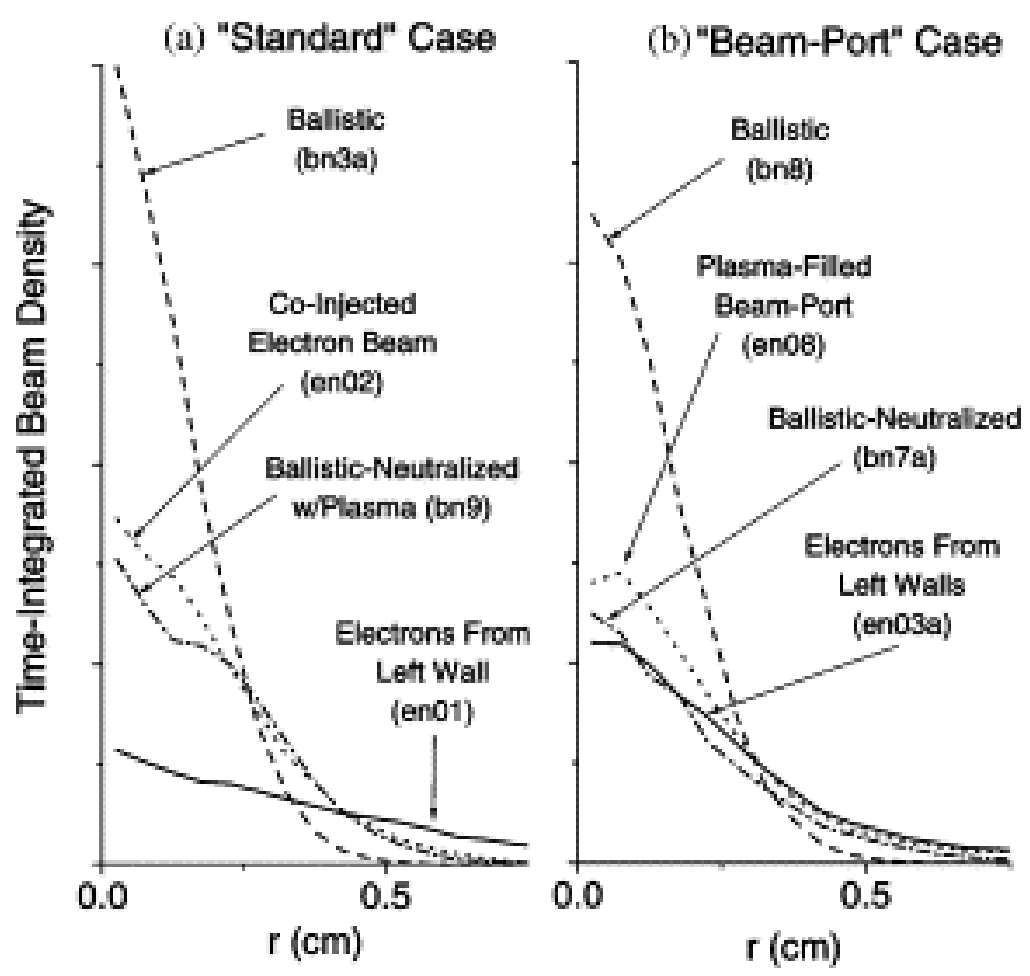

Fig. 6. Time-integrated, normalized radial profile of the beam at $\mathrm{z}=300 \mathrm{~cm}$ for (a) the standard simulation geometry and (b) the beam-port simulation geometry.

\section{Conclusions}

A number of simulations have been carried out to explore the charge and current neutralization of HIF relevant ion beams in a reactor chamber, both gas-filled and evacuated. From the simulations, it is clear that methods of readily supplying electrons to the beam prior to entering the chamber can result in higher degrees of charge and current neutralization. Highly neutralized intense beams can be focused to higher energy densities and may be less likely to experience any deleterious beam-beam interaction effects as multiple beams converge towards the target.

Simulations are presently underway to compare the LSP, BIC [10] and BPIC [6] codes in the ballistic-neutralized transport mode. In addition, models for photo-ionization of the beam and gas by the target are also presently being examined [7 and 11].

Issues that remain to be addressed include possible beam/plasma instabilities and beambeam interactions. As they become available, realistic charge-state-dependent cross- 
sections for beam ion stripping and improved cross-sections for FLiBe ionization will be incorporated into future simulations.

\section{Acknowledgements}

We wish to thank our colleagues from the Beam/Chamber working group for useful discussions.

\section{References}

1. T.P. Hughes, R.E. Clark and S.S. Yu Phys. Rev. ST Accel. Beams 2 (1999), p. 110401.

2. B.G. Logan and D.A. Callahan Nucl. Instr. and Meth. Phys. Res. A 415 (1998), p. 468.

Abstract-Compendex

3. D.A. Callahan-Miller and M. Tabak Nucl. Fusion 39 (1999), p. 883. AbstractCompendex |

Abstract-INSPEC | Full Text via CrossRef

4. D.A. Callahan Fusion Eng. Design 32-33 (1996), p. 441. Abstract | PDF (754 K)

5. N. Barboza Fusion Eng. Design 32-33 (1996), p. 453. Abstract | PDF (788 K)

6. J.L. Vay and C. Deutsch Phys. Plasmas 5 (1998), p. 1190. Abstract-INSPEC | OJPS full text $\mid$ Full

Text via CrossRef

7. W.M. Sharp, D.A. Callahan-Miller and A.B. Langdon Bull. Am. Phys. Soc. 44 (1999), p. 201.

8. D.V. Rose, D.R. Welch, B.V. Oliver, Mission Research Corp. Report No. MRC/ABQ-R-1966, 2000.

9. D.R. Welch, Mission Research Corp. Report No. MRC/ABQ-R-1942, 1999.

10. A.B. Langdon Part. Accel. 37-38 (1992), p. 175. Abstract-INSPEC

11. A.B. Langdon, R.O. Bangerter and D.A. Callahan Nucl. Instr. and Meth. Phys. Res. A 278 (1989), p.

68. Abstract 
*1 Work sponsored by DOE through Lawrence Berkeley National Laboratory. Corresponding author. Tel.: +1-505-768-7786; fax: +1-505-768-7601; email: drose@mrcabq.com 